CARDIOVASCULAR MEDICINE

\title{
Clinical use of multislice spiral computed tomography in 210 highly preselected patients: experience with 4 and 16 slice technology
}

T Beck, C Burgstahler, A Kuettner, A F Kopp, M Heuschmid, C D Claussen, S Schroeder

Heart 2005;91:1423-1427. doi: 10.1136/hrt.2004.049817

See end of article for authors' affiliations

......................

Correspondence to: Dr Priv-Doz Stephen Schroeder, Department of Internal Medicine, Division of Cardiology, OtfriedMueller-Strasse 10, 72076 Tuebingen, Germany; stephen.schroeder@med. uni-tuebingen.de

Accepted 1 December 2004 Published Online First 10 March 2005
Objective: To report an initial experience with multislice spiral computed tomography (MSCT) coronary imaging, as well as differences in diagnostic accuracy between 4 slice and 16 slice MSCT technology. Methods and results: 210 patients underwent MSCT coronary angiography (4 slices, $\mathrm{n}=120 ; 16$ slices, $\mathrm{n}=90$; suspicion of coronary artery disease, $\mathrm{n}=158$; suspicion of restenosis, $\mathrm{n}=52$ ). Recommendations for further diagnostic tests were based on the MSCT results. Patients were interviewed by telephone after a mean (SD) of 449 (169) days to evaluate their further clinical course. MSCT detected significant lesions in 90 of 210 (43\%) patients and invasive coronary angiography (ICA) was recommended. MSCT excluded significant lesions in 120 of $210(57 \%)$ patients. ICA was actually performed in 44 of $210(21 \%)$ patients (corresponding results, 27 of $44(61 \%)$; false positive, 11 of 44 (25\%); false negative, 6 of $44(14 \%))$. No significant differences were found between 4 and 16 slice imaging. No major cardiac event occurred during follow up.

Conclusions: MSCT was found to be useful to evaluate the need for invasive diagnostic procedures. However, the false negative results underline that further improvements of image quality are required before MSCT can replace ICA in carefully selected patients.
$\mathrm{C}$ onventional invasive coronary angiography (ICA) is still the reference standard for the diagnosis of coronary artery disease $(\mathrm{CAD})$ and the detection of coronary artery stenoses. Despite the invasiveness and possible related complications of ICA, the absolute number of ICAs is rising annually, although only about one third of all diagnostic ICAs are followed by percutaneous coronary intervention. ${ }^{12}$ Thus, interest is growing in non-invasive imaging modalities for evaluation of non-invasive diagnosis or exclusion of CAD. ${ }^{3}$

In 1999, multislice spiral computed tomography (MSCT) systems with simultaneous acquisition of four slices and half second scanner rotation became available for non-ICA. Initial experiences have shown that coronary stenoses can be detected with promising sensitivity and specificity. ${ }^{3-8}$

In 2002, the second MSCT scanner generation with faster gantry rotation speed $(370-420 \mathrm{~ms})$ and 16 detector slices became available. A significantly improved image quality led to more accurate detection of coronary artery stenoses. Owing to this technical progress and growing experience of physicians, a sensitivity of $95 \%$ and specificity of $98 \%$ were reported for the detection $>50 \%$ diameter stenoses. ${ }^{9-11}$

Several studies have been published comparing 4 slice and 16 slice MSCT with ICA. However, the use of MSCT has been little reported as the first line imaging technique in clinical practice without additional ICA.

The objective of the present study was to evaluate the usefulness of MSCT coronary angiography as a first line imaging technique to evaluate the need for invasive diagnostic procedures in patients with unclear chest pain because of suspected or progressive CAD after percutaneous transluminal coronary angioplasty (PTCA). Furthermore, differences in image quality, the number of assessable segments, and the difference in diagnostic accuracy between 4 slice and 16 slice MSCT technology were addressed.

\section{METHODS}

We have been examining the heart by MSCT at our institution for more than five years. Because of the high negative predictive value for the detection of coronary stenoses, which was reported in all comparative studies with ICA, MSCT coronary angiography is performed at our cardiac imaging outpatient clinic in routine clinical practice for the exclusion of CAD. ${ }^{4}$

From November 2000 to November 2003, 210 consecutive patients were referred by general practitioners or specialists in internal medicine or cardiology to undergo a non-invasive evaluation of the coronary tree with MSCT. One hundred and twenty patients were assessed by a 4 slice scanner and 90 patients by a 16 slice scanner. All patients were treated with 50-100 mg metoprolol at least 45 minutes before the MSCT scan. MSCT examinations were indicated for patients with clinical suspicion of CAD or of progression of CAD after PTCA or coronary artery bypass graft (CABG) surgery because of chest pain or positive stress tests. Moreover, a smaller number of asymptomatic patients or patients with atypical angina, but a distinctive risk profile for CAD, were examined. Patients with typical angina and positive stress tests were not investigated by MSCT.

Exclusion criteria for MSCT were as follows: acute coronary syndrome, previous stent implantation, chronic congestive heart failure (New York Heart Association functional class III-IV), renal insufficiency (creatinine $>115 \mu \mathrm{mol} / \mathrm{l}$ ), hyperthyroidism (basal thyroid stimulating hormone concentration $<0.1 \mu \mathrm{l} / \mathrm{l}$ ), known allergic reaction to iodated contrast media, chronic obstructive pulmonary disease that prohibited a respiratory stop for 40 seconds, and atrial fibrillation.

Cardiovascular risk factors were defined as follows: smoking, as having smoked at least five cigarettes/day within the previous three months; hyperlipidaemia, as a total serum cholesterol concentration $\geqslant 5.96 \mathrm{mmol} / \mathrm{l}$ or

Abbreviations: $C A B G$, coronary artery bypass graft; $C A D$, coronary artery disease; ICA, invasive coronary angiography; IQS, image quality score; MSCT, multislice spiral computed tomography; PTCA, percutaneous transluminal coronary angioplasty 
serum triglycerides $\geqslant 2.26 \mathrm{mmol} / \mathrm{l}$ or taking lipid lowering medication; hypertension, as having a systolic blood pressure $\geqslant 145 \mathrm{~mm} \mathrm{Hg}$ or diastolic blood pressure $\geqslant 95 \mathrm{~mm} \mathrm{Hg}$ or taking antihypertensive medication; familial predisposition, as having relatives of first or second degree with premature cardiovascular disease; and diabetes mellitus, as the use of oral hypoglycaemic agents or insulin or having increased fasting serum glucose concentrations $(>7.8 \mathrm{mmol} / \mathrm{l})$. Positive stress tests were defined as examinations (exercise ECG, myocardial perfusion imaging) with pathological results.

All patients gave their informed consent for MSCT, systematic anonymous analysis, and publication of the data.

\section{MSCT scanning technique}

One hundred and twenty patients were examined by 4 slice MSCT with a Somatom Volume Zoom scanner (Siemens, Forchheim, Germany) and 90 patients by 16 slice MSCT with a Sensation 16 scanner (Siemens).

The standard scan protocol for MSCT was as follows.

Firstly, a native scan without contrast media was recorded to quantify the total calcium burden of the coronary tree (collimation $1.5 \mathrm{~mm}$, table feed $3.8 \mathrm{~mm} /$ rotation, and tube current 133 effective $\mathrm{mA}$ at $120 \mathrm{kV})$. To evaluate the circulation time, $20 \mathrm{ml}$ of contrast media $(20 \mathrm{ml}$ at $4 \mathrm{ml} / \mathrm{s}$, $400 \mathrm{mg}$ iodine/ml, Iomeron 400 (Altana Pharma, Konstanz, Germany)) and a chaser bolus of $20 \mathrm{ml}$ saline were injected into an antecubital vein. The correct scanning delay was established by measuring computed tomographic attenuation values in the ascending aorta with the first slice after maximum contrast used as circulation time. By using a dual head power injector (CT2, Medtron, Saarbrücken, Germany) a total of $150 \mathrm{ml}$ (4 slice technology) or $80 \mathrm{ml}$ ( 16 slice technology) intravenous contrast agent plus a $20 \mathrm{ml}$ chaser bolus was injected. Computed tomography started at the aortic root cranial to the coronary ostia and stopped at the diaphragm caudally of all cardiac structures.

For image reconstruction, the standard built-in reconstruction algorithm was used. ${ }^{42-14}$ The reconstruction window was set to start at $60 \% \mathrm{RR}$ interval to determine the reconstruction interval with the fewest motion artefacts. If necessary, a test series reconstructing slices ranging from 35$75 \%$ relative to the RR interval was performed in 2\% steps. The time point with the least motion artefacts was then chosen to reconstruct the entire stack of images of the MSCT. Images were reconstructed and data were analysed by an experienced radiologist on an offline workstation for postprocessing.

\section{MSCT image interpretation}

Depending on the individual case, axial slices and advanced post-processing tools, such as multiplanar reconstruction and maximum intensity projection or three dimensional volume rendering techniques, were used. Image quality was determined on contrast media enhanced axial slices for each coronary vessel segment. For documentation, a modified American Heart Association segment model was used (for the right coronary artery, segment 1 is the proximal, 2 is the middle, 3 is the distal, and 4 is the combined posterior descending and posterolateral branches; 5 is the left main stem; for the left anterior descending artery, 6 is the proximal, 7 is the middle, 8 is the distal, 9 is the first diagonal, and 10 is the second diagonal; and for the left circumflex artery, 11 is the proximal, 12 is the distal, and 13 is the marginal branches).

Image quality was graded as 1 (excellent), 2 (good), 3 (still diagnostic), or 4 (not diagnostic) according to the number of evaluable segments. An image quality score (IQS) was calculated as the mean value of all classifications.
On the basis of the results of MSCT and clinical information, patients were grouped and subgrouped as follows: for group I, no coronary angiography was recommended because either (a) coronary arteries were only moderately diseased and without significant stenoses ( $>50 \%$ diameter stenosis) or (b) CAD was excluded (no calcified or non-calcified plaques detectable, Agatston score of 0); and for group II, coronary angiography was recommended because either (a) $a \geqslant 50 \%$ diameter stenosis was detected or (b) insufficient image quality or severe calcifications prohibited accurate determination of lesion severity.

\section{Clinical follow up}

A follow up interview was conducted by telephone to evaluate the further clinical course of our patients. We evaluated (a) whether the referring colleague complied with the recommendation based on the MSCT examinations; (b) the results of coronary angiographies if performed; (c) the agreement between coronary angiography and MSCT results; and $(\mathrm{d})$ the patient's clinical symptoms (graded as l, better; 2, unchanged; or 3, worse).

\section{Statistical analysis}

Continuous variables were described as mean (SD). $t$ Tests and $\chi^{2}$ tests were performed to evaluate differences between 4 and 16 slice results. Values of $p<0.05$ were considered to identify significant differences. All analyses were done with Prism 3.02 (GraphPad Software Inc, San Diego, California, USA).

\section{RESULTS}

Table 1 summarises patient characteristics. Figure 1 shows an image example.

\section{Results of patients who underwent 4 slice MSCT Indication for non-ICA}

Eighty six of $120(72 \%)$ patients had clinical suspicion of CAD, 19 of $120(16 \%)$ patients had the suspicion of progress of their known CAD, and 15 of 120 (12\%) patients had undergone CABG surgery previously. Of these 120 patients, $99(83 \%)$ had angina, $36(30 \%)$ had a pathological exercise ECG, and five $(4 \%)$ had pathological myocardial perfusion imaging.

\section{MSCT image quality}

After image reconstruction, the image quality was graded as excellent for 29 of $120(24 \%)$ patients, as good for $25(21 \%)$, as still diagnostic for 57 (48\%), and as not diagnostic for eight (7\%). The IQS was $2.37(0.93)$ and for each patient 8.23 (2.82) segments were evaluable. The mean Agatston score was 340 (1060) (range 0-8080) (table 2).

\section{Recommendation for further procedures}

In addition to MSCT coronary angiography, an ICA was recommended for 49 of 120 (41\%) patients: for $33(28 \%)$ because severe stenoses were detected and for $16(13 \%)$

Table 1 Clinical characteristics

\begin{tabular}{llll}
\hline & 4 slice MSCT & 16 slice MSCT & p Value* \\
\hline Sex (men/women) & $91 / 29$ & $68 / 22$ & 0.97 \\
Hypertension & $90 / 120(75 \%)$ & $55 / 90(61 \%)$ & 0.35 \\
Diabetes mellitus & $18 / 120(15 \%)$ & $13 / 90(14 \%)$ & 0.92 \\
Smoking & $21 / 120(18 \%)$ & $13 / 90(14 \%)$ & 0.61 \\
High cholesterol & $88 / 120(73 \%)$ & $58 / 90(64 \%)$ & 0.56 \\
Anginat & $99 / 120(83 \%)$ & $55 / 90(61 \%)$ & 0.17 \\
\hline
\end{tabular}

${ }^{*} \chi^{2}$ test; $†$ Canadian Cardiovascular Society classification. MSCT, multislice spiral computed tomography. 

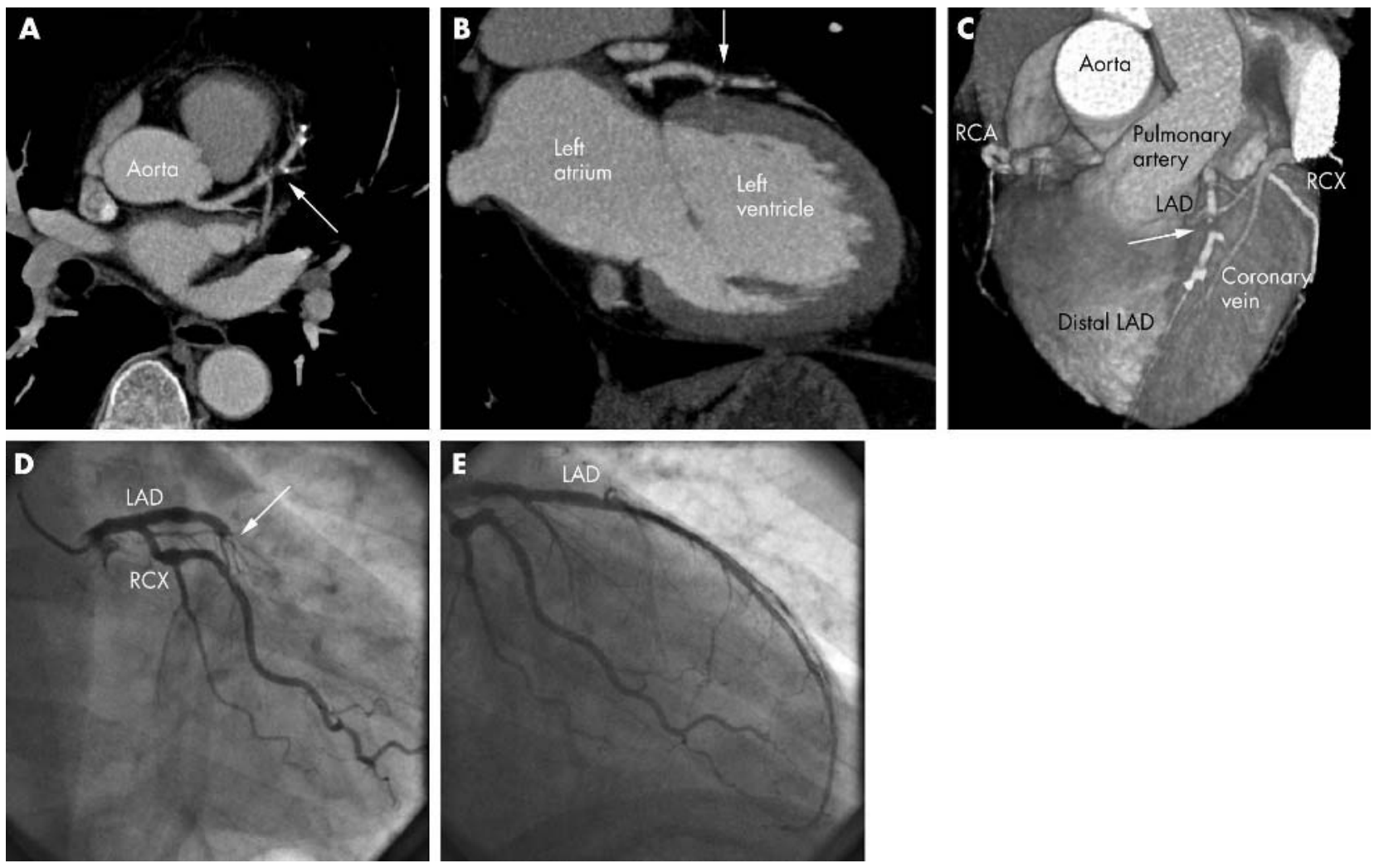

Figure 1 Left coronary artery of an asymptomatic 65 year old male patient. (A) Axial view of the left anterior descending artery (LAD) with mixed plaque in the proximal LAD (arrow). No contrast enhanced lumen is visible within the stenotic area, indicating either a severe subtotal stenosis or chronic total occlusion with retrograde vessel perfusion. (B) Lateral view of the LAD. Mixed plaque with stenotic area (arrow). (C) Three dimensional reconstruction. No contrast enhanced lumen is visible within the plaque area (arrow). After multislice spiral computed tomography (MSCT) coronary angiography was performed the patient was advised to undergo invasive coronary angiography (ICA). (D and E) ICA verified the MSCT findings. The LAD has a proximal chronic occlusion with bridging collaterals (arrow). ICA in right anterior oblique before (D) and after (E) percutaneous coronary intervention. For MSCT coronary angiography it is difficult to differentiate a subtotal stenosis from a chronic occlusion with retrograde vessel perfusion. RCA, right coronary artery; RCX, right circumflex artery.

because segments were unevaluable due to non-diagnostic image quality.

No ICA was recommended for 71 of 120 (59\%) patients: for $46(38 \%)$ because CAD was excluded and for $25(21 \%)$ because severe stenoses were excluded.

\section{Follow up}

Clinical follow up was performed after 16.9 (3.7) months (range 7.5-23.6 months) by telephone interview. Two patients could not be evaluated. One patient died of oesophageal carcinoma and one patient declined to participate in the follow up interview. Twenty six of $120(22 \%)$ underwent ICA. For three patients ICA showed false negative MSCT results with missed coronary stenoses, for 18 patients the results corresponded, and for five patients the results were false positive (table 3 ).

Table 4 summarises symptoms and quality of life at follow up.

Table 2 Image quality of 4 and 16 slice MSCT scanners

\begin{tabular}{llll}
\hline & $\mathbf{4}$ slice MSCT & 16 slice MSCT & p Value* \\
\hline Heart rate (beats $/ \mathrm{min})$ & $65.1(12.4)$ & $62.3(11.0)$ & 0.06 \\
Image quality scoret & $2.4(0.9)$ & $1.7(0.9)$ & $<0.001$ \\
Evaluable segments & $8.2(2.8)$ & $11.1(2.7)$ & $<0.001$ \\
Agatston score & $340.3(1059.5)$ & $421.4(959.5)$ & 0.29 \\
\hline
\end{tabular}

${ }^{*} t$ Test.

†Image quality scored as 1 , excellent; 2 , good; 3 , still diagnostic; 4 , not diagnostic.

Data are mean (SD).

\section{Results of patients who underwent 16 slice MSCT}

Indication for non-ICA

Seventy two of $90(80 \%)$ patients had suspicion of CAD, 12 ( $13 \%)$ had known CAD, and six (7\%) had already undergone CABG surgery. Of these 90 patients, 55 (61\%) had angina, 18 (20\%) had a pathological exercise ECG, and three $(3 \%)$ had pathological myocardial perfusion imaging.

\section{MSCT image quality}

Fifty two of $90(58 \%)$ patients had an image of excellent quality, $21(23 \%)$ of good quality, 11 (12\%) of still diagnostic quality, and six (7\%) of non-diagnostic quality. The IQS was $1.69(0.94)$ and for each patient 11.14 of 2.74 segments were evaluable. The mean (SD) Agatston score was 421 (959) (range 0-6534) (table 2).

\section{Recommendation for further procedures}

An additional ICA was recommended for 33 of 90 (37\%) patients because severe coronary stenoses were detected and for eight ( $9 \%$ ) because of a non-diagnostic image quality. No further invasive diagnostic was recommended for 37 of 90 $(41 \%)$ because severe stenoses was excluded and for 12 ( $13 \%)$ because CAD was completely excluded.

\section{Follow up}

Clinical follow up was performed after 5.1 (1.5) months (range 1.2-6.9 months). No patient was missed by the telephone interview. ICA was actually performed in 18 of 90 $(20 \%)$ of patients. Results corresponded for nine of $18(50 \%)$ 
Table 3 Follow up parameters

\begin{tabular}{|c|c|c|c|c|c|c|c|c|c|}
\hline & \multicolumn{4}{|c|}{4 slice MSCT } & \multicolumn{4}{|c|}{16 slice MSCT } & \multirow[b]{3}{*}{ p value* } \\
\hline & \multicolumn{2}{|c|}{ Group I (n=71) } & \multicolumn{2}{|c|}{ Group II $(n=49)$} & \multicolumn{2}{|c|}{ Group I $(n=49)$} & \multicolumn{2}{|c|}{ Group II $(n=41)$} & \\
\hline & la $(n=46)$ & Ib $(n=25)$ & Ila $(n=33)$ & Ilb $(n=16)$ & la $(n=37)$ & Ib (n= 12) & Ila $(n=33)$ & Ilb (n=8) & \\
\hline ICA recommended & 0 & 0 & 33 & 16 & 0 & 0 & 33 & 8 & 0.67 \\
\hline ICA performed & 4 & 0 & 14 & 8 & 2 & 0 & 16 & 0 & 0.81 \\
\hline \multicolumn{10}{|c|}{ Correspondence of results with MSCT } \\
\hline Yes & 2 & 0 & 9 & 7 & 1 & 0 & 8 & 0 & 0.52 \\
\hline No & $2 \dagger$ & 0 & $5 !$ & $1 \dagger$ & $1 \dagger$ & 0 & $2 \dagger / 6 \ddagger$ & 0 & 0.86 \\
\hline
\end{tabular}

patients, were false negative for three $(17 \%)$, and were false positive for six (33\%) (table 3).

Table 4 summarises symptoms and quality of life.

\section{Comparison of 4 slice and 16 slice results}

Image quality was significantly better with the use of 16 slice technology, which produced significantly more images of coronary segments of diagnostic quality. The diagnostic accuracy was, however, found to be comparable. Table 2 and table 3 summarise the results.

\section{DISCUSSION}

Our data underline that non-invasive MSCT coronary angiography has the potential to reduce the total number of ICAs in carefully selected patient cohorts. However, even with the current 16 slice MSCT technology, we had to recognise a small number of false negative results. These were mainly due to severe calcifications or motion artefacts. Thus, MSCT imaging is useful to rule out significant lesions in case of good image quality and after the exclusion of only severe calcifications.

\section{Multislice spiral computed tomography}

MSCT is a newly emerging non-invasive modality for visualisation of the coronary tree. The high negative predictive values reported by most comparative studies with ICA indicate that MSCT may best be suited for the exclusion of CAD. ${ }^{911}$ However, studies have not evaluated this specific indication and no official guidelines for its clinical use exist.

Our results underline that MSCT may be useful to reduce the number of ICAs in carefully selected patients with a low to intermediate probability of having a severe lesion. All our patients had been referred to our institution by general practitioners for MSCT imaging to evaluate the coronary status by an imaging technique. Without the opportunity of undergoing MSCT imaging, most of these patients would have undergone invasive diagnostic procedures. In our

Table 4 Clinical follow up parameters

\begin{tabular}{|c|c|c|}
\hline & 4 slice MSCT & 16 slice MSCT \\
\hline \multicolumn{3}{|c|}{ Clinical symptoms } \\
\hline Better & $46 / 120(38 \%)$ & $32 / 90(36 \%)$ \\
\hline Unchanged & $69 / 120(58 \%)$ & $54 / 90(60 \%)$ \\
\hline Worse & $3 / 120(3 \%)$ & $4 / 90(4 \%)$ \\
\hline \multicolumn{3}{|l|}{ Quality of life } \\
\hline Better & $41 / 120(34 \%)$ & $31 / 90$ (34\%) \\
\hline Unchanged & $75 / 120(63 \%)$ & $54 / 90(60 \%)$ \\
\hline Worse & $2 / 120(2 \%)$ & $5 / 90(6 \%)$ \\
\hline \multicolumn{3}{|c|}{ Satisfaction with treatment } \\
\hline Very good & $39 / 120(33 \%)$ & $44 / 90(49 \%)$ \\
\hline Good & $77 / 120(64 \%)$ & $34 / 90(38 \%)$ \\
\hline Bad & $2 / 120(2 \%)$ & $12 / 90(13 \%)$ \\
\hline
\end{tabular}

cohort, the total number of ICA was reduced to $21 \%$. MSCT coronary angiography turned out to be a safe procedure with only one observed complication (extravasation of contrast agent in one patient).

\section{Differences between 4 slice and 16 slice MSCT imaging}

One hundred and twenty patients underwent 4 slice MSCT coronary angiography. For each patient 8.2 (2.8) segments were evaluable and the IQS was 2.4 (0.9). In comparison, 90 patients underwent 16 slice MSCT coronary angiography with 11.1 (2.7) evaluable segments for each patient and an IQS of 1.7 (0.9). These results underline an improved image quality in 16 slice scanning because of technical innovations.

However, as table 3 indicates, the better image quality did not lead to improved diagnostic accuracy. In both cohorts, a comparable number of results were false negative. These were mainly due to well known limitations of MSCT scanning: severe calcifications obscured the correct quantification of obviously severe lesions in four patients; and significant lesions were missed because of motion artefacts in two patients. Thus, a native scan before contrast enhancement appears to be meaningful to rule out severe calcifications. In addition, $\beta$ blockade before scanning was found to be useful to lower heart rate. ${ }^{15}$ A recently published study with the latest 16 slice scanner generation with further improved gantry rotation time also reported a small number of false negative results. Those authors also reported that false negative results were related to severe calcifications and motion artefacts. ${ }^{11}$

Further limitations of MSCT imaging have to be taken into account. Since it is a conventional $x$ ray technique, iodinated contrast media are required, prohibiting its use in patients with, for example, hyperthyroidism or renal failure. In addition radiation exposure has to be considered, which is about $5 \mathrm{mSv}$ despite sophisticated software tools with reduced tube current during systole. ${ }^{16} 17$

New scanner generations have been announced with further improved spatial and temporal resolutions. Whether MSCT coronary angiography can be stabilised further to overcome the mentioned limitations remains to be evaluated.

\section{Study limitations}

We report on a clearly preselected patient cohort. The MSCT results were not evaluated by ICA in all cases. Thus, the number of false negative or positive results might have been higher and may have affected the accuracy of the presented data. However, no clinical events occurred during follow up and a decisive number of patients reported improved clinical symptoms.

\section{Conclusions}

Our initial experience with MSCT coronary imaging in clinical practice suggests that it is a feasible and safe method 
for the non-invasive assessment of the coronary system. However, the false negative results underline that further improvements of image quality are required before MSCT can replace ICA in carefully selected patients.

\section{Authors' affiliations}

T Beck*, C Burgstahler*, S Schroeder, Department of Internal Medicine, Division of Cardiology, Eberhard-Karls University, Tuebingen, Germany A Kuettner, A F Kopp, M Heuschmid, C D Claussen, Department of Radiology, Division of Diagnostic Radiology, Eberhard-Karls University, Tuebingen, Germany

*Both authors contributed equally

This study was performed without additional financial support

\section{REFERENCES}

1 Scanlon PJ, Faxon DP, Audet AM, et al. ACC/AHA guidelines for coronary angiography. A report of the American College of Cardiology/American Heart Association task force on practice guidelines (committee on coronary angiography). Developed in collaboration with the Society for Cardiac Angiography and Interventions. J Am Coll Cardiol 1999;33:1756-824.

2 Mannebach H, Hamm C, Horstkotte D. [18th report of the statistics of heart catheter laboratories in Germany. Results of a combined survey by the Committee of Clinical Cardiology and the Interventional Cardiology and Angiology Working Group (for ESC) of the German Society of Cardiology-Heart and Cardiovascular Research 2001]. Z Kardiol 2002;91:727-9.

3 Achenbach S, Daniel WG. Noninvasive coronary angiography: an acceptable alternative? N Engl J Med $2001 ; 345: 1909-10$.

4 Kopp AF, Schroeder S, Kuettner A, et al. Non-invasive coronary angiography with high resolution multidetector-row computed tomography: results in 102 patients. Eur Heart J 2002;23:1714-25.

5 Nieman K, Oudkerk M, Rensing BJ, et al. Coronary angiography with multislice computed tomography. Lancet 2001;357:599-603.
6 Achenbach S, Giesler T, Ropers D, et al. Detection of coronary artery stenoses by contrast-enhanced, retrospectively electrocardiographically-gated, multislice spiral computed tomography. Circulation 2001;103:2535-8.

7 Ohnesorge B, Flohr T, Becker C, et al. Cardiac imaging by means of electrocardiographically gated multisection spiral CT: initial experience. Radiology 2000;217:564-71.

8 Gerber TC, Kuzo RS, Karstaedt N, et al. Current results and new developments of coronary angiography with use of contrast-enhanced computed tomography of the heart. Mayo Clin Proc 2002;77:55-71.

9 Nieman K, Cademartiri F, Lemos PA, et al. Reliable noninvasive coronary angiography with fast submillimeter multislice spiral computed tomography. Circulation 2002;106:2051-4.

10 Ropers D, Baum U, Pohle K, et al. Detection of coronary artery stenoses with thin-slice multi-detector row spiral computed tomography and multiplanar reconstruction. Circulation 2003;107:664-6.

11 Mollet NR, Cademartiri F, Nieman K, et al. Multislice spiral computed tomography coronary angiography in patients with stable angina pectoris. J Am Coll Cardiol 2004;43:2265-70.

12 Flohr T, Bruder H, Stierstorfer K, et al. New technical developments in multislice CT, part 2: sub-millimeter 16-slice scanning and increased gantry rotation speed for cardiac imaging. Rofo Fortschr Geb Rontgenstr Neven Bildgeb Verfahr 2002; 174:1022-7.

13 Heuschmid M, Kuttner A, Flohr T, et al. [Visualization of coronary arteries in $C T$ as assessed by a new 16 slice technology and reduced gantry rotation time: first experiences]. Rofo Fortschr Geb Rontgenstr Neuen Bildgeb Verfahr 2002;174:721-4

14 Kopp AF, Ohnesorge B, Flohr T, et al. [Cardiac multidetector-row CT: first clinical results of retrospectively ECG-gated spiral with optimized temporal and spatial resolution]. Rofo Fortschr Geb Rontgenstr Neven Bildgeb Verfahr 2000;172:429-35.

15 Schroeder S, Kopp AF, Kuettner A, et al. Influence of heart rate on vessel visibility in noninvasive coronary angiography using new multislice computed tomography: experience in 94 patients. Clin Imaging 2002;26:106-11.

16 Becker C, Schatzl M, Feist $H$, et al. [Assessment of the effective dose for routine protocols in conventional CT, electron beam CT and coronary angiography]. Rofo Fortschr Geb Rontgenstr Neven Bildgeb Verfahr 1999:170:99-104.

17 Jakobs TF, Becker CR, Ohnesorge B, et al. Multislice helical CT of the heart with retrospective ECG gating: reduction of radiation exposure by ECGcontrolled tube current modulation. Eur Radiol 2002;12:1081-6.

\section{IMAGES IN CARDIOLOGY}

\section{Biventricular pacemaker implant in a patient with persistent left sided superior vena cava}

$\mathrm{P}$ ersistence of a left sided superior vena cava (SVC) is the most common thoracic venous congenital anomaly. It results from obliteration of the proximal part of the right anterior and the right common cardinal veins. The left anterior cardinal vein persists, forming a left sided vena cava which drains into the coronary sinus.

A 38 year old woman with a history of idiopathic dilated cardiomyopathy and left bundle branch block underwent placement of a biventricular pacemaker. She was not previously known to have a left sided SVC which only became apparent when, after cannulation of the left subclavian vein, the guidewire passed along the left side of the mediastinum. The image shows a postero-anterior film of the implanted device and leads.

The presence of a left sided SVC is appreciated. The three leads enter the venous circulation via the left subclavian vein and pass through the left sided superior vena cava and the dilated coronary sinus to enter the right atrium (a), right ventricle (b), and a posterolateral tributary of the coronary sinus (c).

A persistent left sided SVC poses technical challenges in the placement of the left ventricular pacing lead as the usefulness of coronary sinus venography is limited by inability to occlude the relatively large vein. The condition of this patient improved following device implantation and she remains well and pacing thresholds are stable at one year follow up.

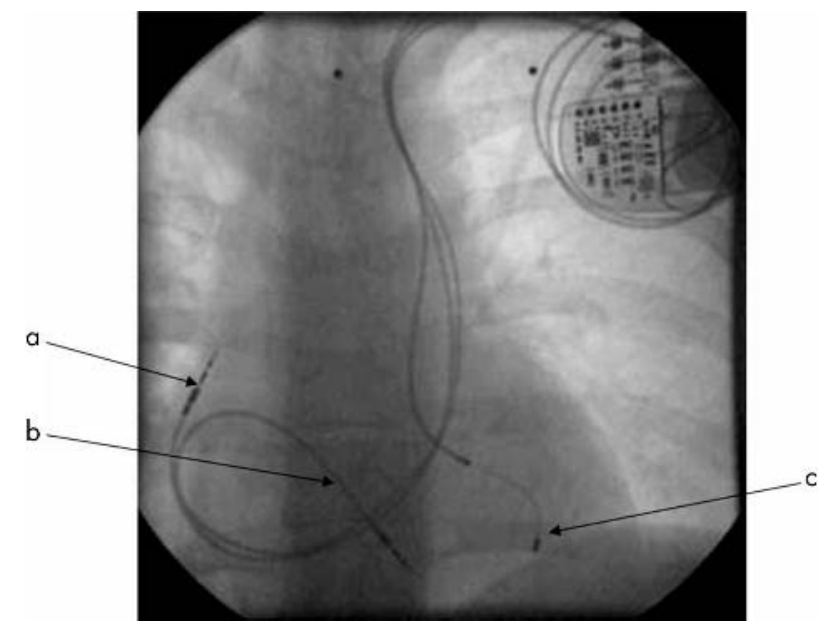

Y M Smyth

C D Barrett

G J Fahy

ysmyth@yahoo.com 